Cite this: RSC Adv., 2013, 3, 22261

Received 15th July 2013

Accepted 17th September 2013

DOI: 10.1039/c3ra43622e

www.rsc.org/advances

\section{Electrospun amino-functionalized PDMS as a novel SPME sorbent for the speciation of inorganic and organometallic arsenic species $\uparrow$}

\author{
Ezel Boyac1,ł Nesrin Horzum, \$ Ali Çağır, Mustafa M. Demir $\S^{*}$ and Ahmet E. Eroğlu* \\ Sol-gel based amine-functionalized SPME fibers (PDMS-weak anion exchanger) were prepared and used \\ for direct mode extraction of dimethylarsinic acid (DMA), monomethylarsonic acid (MMA), and arsenate \\ $(\mathrm{As}(\mathrm{V}))$ from aqueous solutions followed by HPLC-ICPMS determination. Two different methods of \\ coating were employed: (i) electrospinning and (ii) dip coating. Electrospinning was used for the first \\ time for preparation of sol-gel based SPME fibers and was found to be superior in terms of extracted \\ amount of arsenicals, coating homogeneity, accessibility of amine groups on the surface, and \\ preparation time for a single fiber. Various parameters such as solution $\mathrm{pH}$, extraction time, agitation \\ speed, extraction temperature, and ionic strength were studied. Optimum extraction conditions were \\ determined as $\mathrm{pH}$ of 5.0, extraction time of $30 \mathrm{~min}$, agitation speed of $700 \mathrm{rpm}$, and extraction \\ temperature of $20{ }^{\circ} \mathrm{C}$. Extraction ability of the novel coating decreased by the addition of $\mathrm{NaCl}$ as a \\ consequence of the competition between anionic arsenic species and chloride ions for active sites of the \\ weak anion exchanger. This novel sol-gel coating prepared by electrospinning was found to be \\ promising for SPME applications. Vibrational spectroscopy revealed the alignment of PDMS chains by \\ elongational force under electrospinning process. The chain alignment accordingly orients the pendant \\ amino functional groups perpendicular to the fiber surface, which may develop the free active \\ functional groups available to the medium and lead to the enhancement of the extraction \\ performance. Moreover, the proposed coating strategy through electrospinning might be able to break \\ new ground for various applications in analytical chemistry as well as other disciplines.
}

\section{Introduction}

Sample preparation is one of the most critical and challenging steps in a chemical analysis owing to complex nature of the matrix for most of the environmental and biological samples. From the analytical point of view, it can help to isolate the analyte from sample matrix, preconcentrate or reconstitute in a more appropriate solvent and give a prospect for reliable trace level determination of analytes. In addition to being timeconsuming and tedious, the entire analytical process can even fail unless an appropriate sample preparation method is used. For instance, one of the commonly used sample preparation method - conventional liquid-liquid extraction (LLE) - requires high amount of samples and large volume of organic solvents;

Department of Chemistry, İzmir Institute of Technology, Urla, 35430, İzmir, Turkey. E-mail: ahmeteroglu@iyte.edu.tr

$\dagger$ Electronic supporting information (ESI) available: Operation conditions for HPLC-ICPMS, chromatograms in the presence and absence of the collision gas, effect of solution $\mathrm{pH}$, agitation time and speed, $\mathrm{NaCl}$ concentration, solution temperature on extraction of arsenic species. See DOI: 10.1039/c3ra43622e

$\$$ These authors contributed equally to the work.

$\S$ Also at: Department of Materials Science and Engineering, İzmir Institute of Technology, 35430, İzmir, Urla, Turkey. E-mail: mdemir@iyte.edu.tr. hence it gives place to sorbent-based techniques such as solid phase extraction (SPE) and solid phase microextraction (SPME). Recent approaches in these techniques provide advantages thanks to the availability of a variety of commercial products and sorbent materials such as inorganic oxides like silica, carbon, and polymers. During the last two decades, the development of some miniaturized extraction techniques originated from solid phase extraction (SPE) has allowed simplified sample preparation and onsite sampling with subsequent instrumental analysis. However, SPE has limitations due to its difficulty in method development, not applicable for onsite sampling, greater complexity as well as cost resulting from being single use. ${ }^{1}$ Solid-phase microextraction (SPME) methodology is a feasible alternative to LLE and SPE. It has been frequently used owing to its ease of operation, being rapid, portable and solventfree. This methodology enables developing various fiber coatings with both advanced extraction efficiency and selectivity. SPME fiber consists of a fused silica core coated with an active layer. The promising commercially available coatings are polydimethylsiloxane (PDMS) for nonpolar, polydimethylsiloxane/ divinylbenzene (PDMS/DVB) for semi-polar, polyacrylate (PA) and carbowax/divinylbenzene (CW/DVB) for polar compounds. ${ }^{2}$ The studies related to production of the new fiber coatings are 
extended to develop solvent-resistive and thermally stable active coatings as well as to enhance mechanical strength of the phase.

Recently reviewed fiber coating processes involve sol-gel methods, ${ }^{3-5}$ molecular imprinting, ${ }^{6,7}$ electrochemical methods, ${ }^{8,9}$ adsorption of polymeric ionic liquids, ${ }^{\mathbf{1 0 - 1 2}}$ and immobilization of nano and micro sized functional particles. Among these methods, sol-gel method offers the opportunity for attachment of various functionalities to the coating which expands the working area of SPME. Various approaches have been reviewed in literature for immobilization of the aforementioned active phases on a SPME fiber. These approaches are dipping the fiber into the solution of active phase, spraying, brushing and electrospinning of the solution/slurry onto fiber. ${ }^{\mathbf{1 3}}$ Among these coating methods, electrospinning has the ability to fabricate micro/nanofibers, to control morphology and for orientation of nanofibrous mat. ${ }^{\mathbf{1 4 - 1 6}}$ The applicability of electrospun fibers as SPME sorbent for the extraction of both nonpolar and polar compounds was reported by Zewe et al. ${ }^{17}$ and it was found that the electrospun polymeric negative photoresist, SU-8 2100, fiber coated wires displayed the best performance with low detection limits and large linear ranges for phenolic compounds. Bagheri and Aghakhani evaluated polyurethane and polyaniline-nylon-6 electrospun fibers for the extraction of chlorobenzenes. ${ }^{\mathbf{1 8 , 1 9}}$ Similarly, Bagheri et al. suggested polyamide fiber coating for the extraction of chlorophenols ${ }^{20}$ from aqueous systems. As described above, the current electrospun SPME fiber coatings are based on electrospinnable polymers. Generally, these polymers suffer from low thermal stabilities which limit their application areas.

Herein, we present, for the first time to the best of our knowledge, a novel method for SPME fiber coating by combination of both sol-gel chemistry and electrospinning. The solgel solution was tailored by blending PDMS and 3-(triethoxysilyl) propylamine (APTES). PDMS has unique physical and chemical properties resulting from the nature of the siloxane backbone and methyl groups attached to silicon. Due to its nontoxicity, low chemical reactivity, thermal and mechanical stability, and high surface activity, PDMS is used in a wide variety of application areas such as microfluidic devices, biomedical products, personal, household, and automotive care industry. ${ }^{21-24}$ PDMS can be functionalized using APTES which has terminal amine groups providing sorption sites for arsenic species. The amine-functionalized PDMS electrospun film coated SPME fibers were utilized for the trace determination of arsenic species to survey their extraction feasibility. There are numerous reasons to select the arsenic as a model analyte in this study. First, arsenic is a ubiquitous element which is present in the atmosphere, groundwater, soil, rocks, and organisms. Second, most of the arsenic compounds are known to be toxic; although their toxicity depends on the oxidation state and the order of methylation of particular species. Therefore, it is important to determine individual species in a sample rather than the total concentration. Finally, most of the organometallic and inorganic arsenic species are anionic in aqueous systems which enable using weak anion exchange capability of novel coating for their determination. The proposed SPME-HPLC-ICPMS method was found to be promising for the individual determination of the arsenicals.

\section{Experimental}

\subsection{Reagents and solutions}

All the chemicals were of analytical reagent grade. Ultrapure water $(18.2 \mathrm{M} \Omega \mathrm{cm})$ was used throughout the study. Glassware and stirring bars were cleaned by being soaked in $10 \%(\mathrm{v} / \mathrm{v})$ nitric acid for $24 \mathrm{~h}$ and rinsed with ultrapure water prior to use.

Stock standard solutions of $\mathrm{As}(\mathrm{v}), 2000.0 \mathrm{mg} \mathrm{L} \mathrm{L}^{-1}$, were prepared by dissolving $\mathrm{As}_{2} \mathrm{O}_{5}$ (Merck, product code: 1.09939, CAS no.: [1303-28-2]) in $1.0 \%$ (v/v) HCl. Dimethylarsinic acid

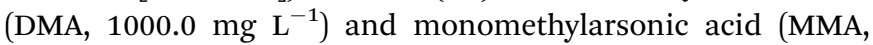
$553.0 \mathrm{mg} \mathrm{L}^{-1}$ ) were prepared by dissolving dimethylarsinic acid sodium salt trihydrate (Merck, product code: 8.20670 , CAS no.: [6131-99-3]) and disodium methyl arsenate hexahydrate (Supelco, product no.: PS 281, CAS no.: [144-21-8]), respectively in ultrapure water. The concentrations of all the stock solutions described above were given in terms of arsenic. Prepared stock solutions were stored in refrigerator at $4{ }^{\circ} \mathrm{C}$; lower concentrations were prepared daily by diluting appropriate amount of stocks in ultrapure water. $\mathrm{HNO}_{3}$ (Merck, product code: 1.00456, CAS no.: [7697-37-2]) and $\mathrm{NH}_{3}$ (Merck, product code: 1.05422 ) were used to adjust the $\mathrm{pH}$ of the solutions.

For HPLC-ICPMS studies, $10.0 \mathrm{mM}$ and $30.0 \mathrm{mM}\left(\mathrm{NH}_{4}\right)_{2} \mathrm{CO}_{3}$ solutions ( $\mathrm{pH}$ 8.50) were freshly prepared by dissolving a proper amount of HPLC grade $\left(\mathrm{NH}_{4}\right)_{2} \mathrm{CO}_{3}$ (Fluka, product code: 74415 , CAS no.: [506-87-6]) in ultrapure water, and pHs of the solutions were adjusted by using various concentrations of $\mathrm{HNO}_{3}$ and $\mathrm{NH}_{3}$.

All solutions including mobile phase and eluting solvent were filtered through $0.20 \mu \mathrm{m}$ cellulose acetate filter paper (Sartorius) and degassed for $15 \mathrm{~min}$ in an ultrasonic bath before being used in the chromatographic column.

Fiber optic cable was kindly supplied by HES Kablo (Kayseri, Turkey). Acetone (Merck, product code: 1.00014, CAS no.: [6764-1]) was used for the removal of protective polyamide coating of the fibers. The introduction of high number of silanol groups on the surface of the bare fiber was achieved by the treatment with $1.0 \mathrm{M} \mathrm{NaOH}$ solution prepared by using $\mathrm{NaOH}$ pellets (Merck, product code: 1.06498, CAS no.: [1310-73-2]). $0.1 \mathrm{M} \mathrm{HCl}$ (Merck, product code: 1.00314, CAS no.: [7647-01-0]) was used for neutralization of the base.

Polydimethylsiloxane, PDMS (hydroxyl terminated, typical viscosity: 25 cSt), (Aldrich, product code: 481939, CAS no.: [70131-67-8]) was used as organic polymer with sol-gel active terminal. Tetrahydrofuran, THF (Sigma Aldrich, product code: 87368, CAS no.: [109-99-9]) was used as solvent in sol-gel process. 3-(Triethoxysilyl) propylamine, APTES (Merck, product code: 8.26619, CAS no.: [14814-09-6]) was used as sol-gel active silane coupling reagent with amine functionality. Trifluoroacetic acid, TFA (Merck, product code: 76-05-1, CAS no.: [808260]) was used as a catalyst in sol-gel process.

\subsection{Instrumentation and apparatus}

The separation of extracted arsenic species was achieved with Agilent 1200 Series HPLC system with a $250 \mathrm{~mm}$ anion exchange column (Hamilton, PRP-X100). Gradients with $10.0 \mathrm{mM}$ and $30.0 \mathrm{mM}\left(\mathrm{NH}_{4}\right)_{2} \mathrm{CO}_{3}$ solutions $(\mathrm{pH} 8.50)$ were used as mobile 
phase at a flow rate of $1.0 \mathrm{~mL} \mathrm{~min}^{-1}$. Chromatographic conditions were $10.0 \mathrm{mM}\left(\mathrm{NH}_{4}\right)_{2} \mathrm{CO}_{3}$ for the first $4 \mathrm{~min}, 30.0 \mathrm{mM}$ $\left(\mathrm{NH}_{4}\right)_{2} \mathrm{CO}_{3}$ for 4-13 min and finally $10.0 \mathrm{mM}\left(\mathrm{NH}_{4}\right)_{2} \mathrm{CO}_{3}$ for 2 min for preconditioning of column. $\mathrm{pH}$ of the solutions was adjusted with 0.01-1.0 $\mathrm{M} \mathrm{HNO}_{3}$ and 0.01-1.0 $\mathrm{M} \mathrm{NH}_{3}$ using Ino Lab Level $1 \mathrm{pH}$ meter (Weilheim, Germany). Detection of each species was realized with ICP-MS, Agilent 7500ce (Tokyo, Japan). Online separation and detection of arsenic species were accomplished by connecting the HPLC column outlet directly to ICP-MS concentric nebulizer. Two instruments were connected by the shortest possible length $(67 \mathrm{~cm})$ of perfluoroalkoxy (PFA) tubing (ID: $0.5 \mathrm{~mm}$ ) to prevent broadening of the peaks. The operating parameters for HPLC and ICP-MS are provided in Table S1 in the ESI. $\dagger$ Extraction of arsenic species was performed in multi-position magnetic stirrer RO 10 power IKAMAG.

The morphology of bare and coated silica fibers was examined using a Philips XL-30SFEG scanning electron microscope (SEM) (Eindhoven, The Netherlands). In electrospinning process, the syringe was connected to a high voltage power supply (Gamma High Voltage Research, Ormond Beach, FL, USA) and fixed on the microsyringe pump (LION WZ-50C6). Fourier Transform Infrared (FTIR) spectra were registered in a Perkin Elmer Spectrum 100 spectrometer with a spectral resolution of $4 \mathrm{~cm}^{-1}$. Polarization of the incident IR radiation was achieved with a ZnSe polarizer. To evaluate the possible orientation of functional groups provided by electrospinning process, the prepared sol-gel solution was directly electrospun onto the $\mathrm{KBr}$ pellets. In comparison to electrospun coated sample, the sol-gel solution was drop-casted onto the $\mathrm{KBr}$ pellets.

\subsection{Preparation of the SPME fibers}

The protective polyamide layer of the silica fiber was removed by immersing the piece of $7 \mathrm{~cm}$ cut into acetone. The bare fibers (diameter of $\sim 125 \mu \mathrm{m}$ ) were washed first with ultrapure water. Introduction of maximum amount of silanol groups on bare silicate surface required for coupling reaction was accomplished by dipping segments of the bare silicate fibers into $1 \mathrm{M}$ $\mathrm{NaOH}$ solution for $60 \mathrm{~min}$. This step was followed by dipping the fiber into $0.1 \mathrm{M} \mathrm{HCl}$ solution for $30 \mathrm{~min}$ to neutralize the excess base. Fibers were washed with ultrapure water and thermal curing was applied for $24 \mathrm{~h}$ at $120^{\circ} \mathrm{C}$.

The sol-gel solution used for coating SPME fibers was prepared by end-functionalization of hydroxyl terminated chains of PDMS with APTES. In a typical procedure, $2.0 \mathrm{~mL}$ PDMS, $3.0 \mathrm{~mL}$ THF and $1.0 \mathrm{~mL}$ APTES were mixed in a $20 \mathrm{~mL}$ vial. Subsequently, $1.0 \mathrm{~mL}$ of TFA $\left(95 \mathrm{v}\right.$ TFA $\left.+5 \mathrm{v} \mathrm{H}_{2} \mathrm{O}\right)$ was added into the solution, mixed thoroughly, and stirred for $48 \mathrm{~h}$ at room temperature. Electrospinnable sol-gel solution was formed by adjusting the viscosity of the solution through evaporation of the solvent at $80{ }^{\circ} \mathrm{C}$ for $2 \mathrm{~h}$. The resulting sol-gel solution was loaded into a syringe and electrospinning process was performed at $25 \mathrm{kV}$ potential difference with the feeding rate of $5.0 \mathrm{~mL} \mathrm{~h}^{-1}$. The distance between the syringe and the metal collector was $10 \mathrm{~cm}$. In order to obtain a homogeneous coating
Table 1 Extraction parameters and ranges used throughout the study ${ }^{a}$

\begin{tabular}{ll}
\hline Parameter & Range \\
\hline pH of solution & $3.0,5.0,7.0,9.0$ \\
Agitation time $(\mathrm{min})$ & $5,15,30,60,120$ \\
Agitation speed $(\mathrm{rpm})$ & $300,500,700,1000$ \\
Concentration of salt $(\mathrm{M})$ & $0,0.01,0.10,1.0$ \\
Solution temperature $\left({ }^{\circ} \mathrm{C}\right)$ & $20,35,55$ \\
${ }^{a}$ Sample volume and arsenic concentrations are kept constant as 15.0 \\
$\mathrm{~mL}$ and $10.0 \mu \mathrm{g} \mathrm{L}{ }^{-1}$, respectively.
\end{tabular}

on the fiber, the plain silica fiber was attached on a rotating drum (300 rpm) which was placed between the metal collector and syringe ( $3 \mathrm{~cm}$ away from the syringe). During the process $2 \mathrm{~cm}$ of the fiber was coated. Coated fibers were conditioned at $110{ }^{\circ} \mathrm{C}$ overnight.

SPME fibers were also prepared by dip coating using the above mentioned sol-gel solution. $200 \mu \mathrm{L}$ disposable pipette tip was blocked and used as a container for sol-gel solution. The bare activated fiber was immersed $(2 \mathrm{~cm})$ in the coating solution and allowed to react for $30 \mathrm{~min}$. Then the fiber was removed with constant speed of $1.0 \mathrm{~mm} \mathrm{~min}^{-1}$ and conditioned at $110^{\circ} \mathrm{C}$ overnight.

\subsection{Extraction of the arsenic species}

Extractions were performed in a $20 \mathrm{~mL}$ vial containing $15.0 \mathrm{~mL}$ of aqueous arsenic mixture solution (DMA, MMA and As(v)) which was stirred with a magnetic bar. Desorption of the analytes was carried out in a container which was prepared from $200 \mu \mathrm{L}$ pipette tip blocked at the base by a gentle application of heat. Various desorption solutions were tested and $\mathrm{KH}_{2} \mathrm{PO}_{4}$ was chosen as a proper eluent. Optimized desorption conditions were; desorption time of $20 \mathrm{~min}$, desorption volume of $150 \mu \mathrm{L}$, eluent concentration of $50.0 \mathrm{mM} \mathrm{KH}_{2} \mathrm{PO}_{4}$ (results are not shown). Extraction parameters such as extraction $\mathrm{pH}$, extraction time, agitation (stirring) speed, ionic strength and extraction temperature were optimized. The investigated parameters and ranges are listed in Table 1.

\section{Results and discussion}

\subsection{Characterization of prepared SPME fibers}

In this study, the main strategy was to prepare a sol-gel based coating for solid phase microextraction of various arsenicals from water. For this purpose, first of all, a sol-gel solution containing PDMS and APTES was prepared. In dip coating, which is the most common method used for sol-gel based coating of SPME fibers, the bare fiber is dipped into a sol-gel solution for a pre-determined time and then the fiber is removed at a constant speed to obtain homogeneous coating. In addition to dip coating, this study reports, for the first time, an alternative method for immobilization which is fundamentally depending on electrospinning of the sol-gel solution on the SPME fiber surface. An electrospinning time of $12 \mathrm{~s}$ was selected to achieve homogeneous coating on the surface of the fiber 
which provided also comparable thicknesses on final coatings from both of the aforementioned methods.

SEM micrographs of amino-functionalized PDMS coated fibers by dip-coating and electrospinning are shown in Fig. 1. The diameters of the fibers after coating processes were $138 \pm 0.5 \mu \mathrm{m}$ and $130 \pm 0.5 \mu \mathrm{m}$ for dip coated and electrospun coated fibers respectively. The subtraction of the diameter of bare SPME fiber $(\sim 125 \mu \mathrm{m})$ from the final diameter gives an idea about the thickness of the coated film, which was about $6.5 \mu \mathrm{m}$ for dip coated and $2.5 \mu \mathrm{m}$ for electrospun coated fibers. In order to obtain thicker coating by electrospinning method various electrospinning times were tested. However, by application of longer spinning times homogeneity was lost and mainly bulb like accumulation of the coating material over the fiber was observed owing to the visco-elastic behavior of the final sol-gel solution.

Uniaxially aligned fibers were obtained using a special geometry of counter electrodes. Two metal strips were positioned parallel to each other with an air gap (nearly $1 \mathrm{~mm}$ ). The electrical field lines are forced to the edge of the strips and the fibers are obtained in between the metal strips. Polarized FTIR spectroscopy was used to investigate molecular orientation of aminofunctionalized PDMS under electrical field. Fig. 2 presents the polarized FTIR spectra of the coating film prepared by dropcasting and electrospinning. The spectra of PDMS ${ }^{25}$ and APTES ${ }^{26}$ are in line with the literature. Parallel and perpendicularly polarized spectra of drop-casted film are found to be analogous, indicating that there is no preferential alignment of PDMS chains (Fig. 2a). However, for the electrospun coated film, a remarkable difference was observed depending on the polarization of the incident IR beam (Fig. 2b). Upon polarization of incident IR beam, the intensities of the vibrational bands undergo change although they appear at the same wavenumbers. For instance, the vibrational bands at $1680 \mathrm{~cm}^{-1}$ and $800 \mathrm{~cm}^{-1}$ which correspond to $\mathrm{N}-\mathrm{H}$ bending and $\mathrm{Si}-\mathrm{O}$ symmetrical stretching were taken into consideration. When the incident IR beam is parallelpolarized to the electrospun amine-functionalized PDMS film axis, the absorbance of $\mathrm{Si}-\mathrm{O}$ bond vibration is higher and $\mathrm{N}-\mathrm{H}$ bond vibration is lower. The comparison between the absorbance of the backbone and the amine groups which are perpendicular to the backbone, gives valuable insight into the segmental orientation of $\mathrm{Si}-\mathrm{O}$ chains in the elongation direction of the film. The degree of uniaxial orientation of polymer chains can be expressed by the dichroic ratio (R). ${ }^{27}$ The dichroic ratio is
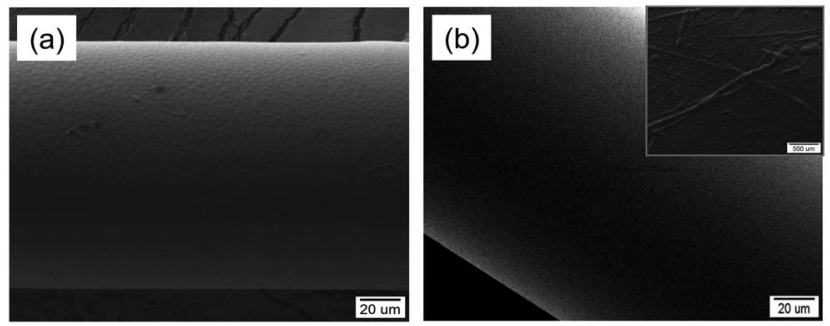

Fig. 1 SEM micrographs of amino-functionalized PDMS (a) dip coated, and (b) electrospun film coated SPME fibers.

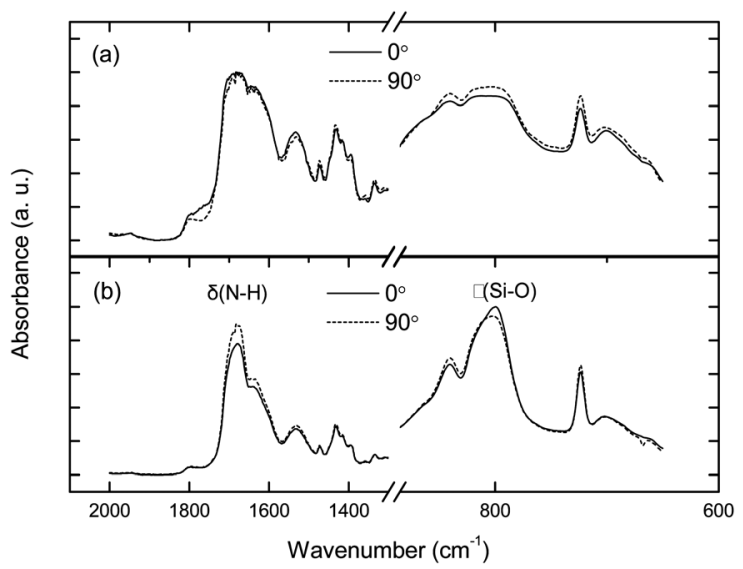

Fig. 2 Polarized FTIR spectra of (a) drop-coated and (b) electrospun aminofunctionalized PDMS film coated $\mathrm{KBr}$ pellets.

calculated by the formula $R=A_{\|} / A_{\perp}$, where $A_{\|}$and $A_{\perp}$ refer to parallel and perpendicularly polarized absorbance intensities for a particular vibration, respectively. The ratio for the bands at 800 and $1680 \mathrm{~cm}^{-1}$ is 0.9 and 1.2 respectively. One can propose that the high extensional force caused by electrospinning process provides the functional amine groups to be more aligned normal to the film surface.

It has to be noted that PDMS has high molecular elasticity due to the flexible Si-O bond, ${ }^{28}$ so that PDMS chains have tendency to recover to their original positions upon electrical force perturbation. However, chain alignment with higher dichroic ratio has been shown for electrospun fibers with various chemistries ${ }^{27,29}$ in literature. On the other hand, the vibration signal at $1430 \mathrm{~cm}^{-1}$ originates from $\mathrm{C}-\mathrm{H}$ bonds and the band centered at $720 \mathrm{~cm}^{-1}$ is attributed to the $\mathrm{Si}-\mathrm{O}-\mathrm{Si}$ bond.

\subsection{Optimization of chromatographic parameters for HPLC- ICPMS}

Most of the buffers used in the chromatographic separation of arsenic species result in salt deposition on skimmer and sample cones of the ICP-MS. To prevent the formation of such depositions, $\left(\mathrm{NH}_{4}\right)_{2} \mathrm{CO}_{3}$ solution was chosen as a proper eluent for chromatographic speciation. The effect of flow rate of mobile phase and addition of an organic modifier such as 1 and $2 \%$ $(\mathrm{v} / \mathrm{v})$ methanol into the buffer solutions as well as various concentrations of $\left(\mathrm{NH}_{4}\right)_{2} \mathrm{CO}_{3}$ were evaluated for optimization of chromatographic conditions in terms of peak intensity, separation and peak symmetry (results are not shown). In the final method, 10.0 and $30.0 \mathrm{mM}\left(\mathrm{NH}_{4}\right)_{2} \mathrm{CO}_{3}$ (pH adjusted to 8.50)

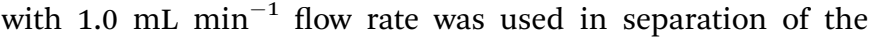
species. The elution order of the species from the strong anion exchange column is DMA, MMA and As(v). The retention mechanism of the species was strongly controlled by the $\mathrm{pH}$ of the mobile phase and dissociation of acidic species. The $\mathrm{pH}$ dependent dissociations of arsenic species are given in Table 2. Depending on the ionic character of the column and the $\mathrm{pH}$ of the eluting buffer ( $\mathrm{pH}$ 8.50), it can be suggested that each arsenic species is eluted in accordance with its charge. 
Table 2 Arsenic species used throughout the study

\begin{tabular}{|c|c|c|c|}
\hline As species & Structure & $\mathrm{p} K_{\mathrm{a}}$ & Reference \\
\hline Arsenate As(v) & $\|_{\mathrm{OH}}^{\mathrm{OH}}-\mathrm{OH}$ & $\begin{array}{r}2.3 \\
6.8 \\
11.6\end{array}$ & 30 \\
\hline $\begin{array}{l}\text { Monomethylarsonic } \\
\text { acid MMA }\end{array}$ & $\|_{\mathrm{OH}} \mathrm{CH}-\mathrm{OH}$ & $\begin{array}{l}3.6 \\
8.2\end{array}$ & 31 \\
\hline $\begin{array}{l}\text { Dimethylarsinic } \\
\text { acid DMA }\end{array}$ & $\|_{\mathrm{CH}_{3}} \mathrm{C}-\stackrel{\mathrm{Os}}{\|_{\mathrm{H}_{3}}} \mathrm{OH}$ & 6.3 & 31 \\
\hline
\end{tabular}

The most common interference regarding the determination of arsenical by ICP-MS is potential polyatomic interference due to the formation of $\mathrm{Ar}^{40} \mathrm{Cl}^{35}$ in the plasma at the same $\mathrm{m} / \mathrm{z}: 75$ during the measurement of monoistopic As ${ }^{75}$ by HPLC-ICPMS. In this study, the octopole collision cell of the ICP-MS system was used with $\mathrm{He}$ as the collision gas to overcome the potential interference of chloride ion on arsenic signal. In addition, the elution peak of $\mathrm{Cl}^{-}$ion was determined by injection of $150 \mu \mathrm{L}$, $0.010 \mathrm{M} \mathrm{NaCl}$ solution into HPLC-ICPMS (Fig. S1†) under optimized chromatographic run conditions in presence and absence of collision gas. The $\mathrm{Cl}^{-}$ion peak was appeared around $500 \mathrm{~s}$ in the chromatograms obtained at m/z: 37 (Fig. S1(a) and (c) $\dagger$ ) both in absence and presence of collision gas, respectively. Chromatogram acquired at $m / z$ : 75 (Fig. S1(b) $\dagger$ ) shows a small peak related to $\mathrm{Ar}^{40} \mathrm{Cl}^{35}$ which was eliminated by addition of $\mathrm{He}$ gas into octopole collision cell (Fig. S1(d)†).

\subsection{Optimization of fiber coating}

The amine-functionalized PDMS coated SPME fibers prepared by both dip-coating and electrospinning were used for the extraction of arsenicals. The results were evaluated for comparison of the coating methods in terms of extraction performances. The variation of amine content in sol-gel solution was also considered in the same study. Based on the results (Fig. 3), a significant difference in extraction of arsenicals was observed between these methods, and superior extraction properties were obtained by electrospun film coated fibers. Under the consideration of the comparable coating thickness and sol-gel solution, the only difference between these two methods is the application of the electric field in the electrospinning method. A typical chromatogram obtained after application of proposed method (SPME-HPLC-ICPMS) is shown in Fig. 4. The extraction was performed with electrospun coated fibers from water containing $10.0 \mu \mathrm{g} \mathrm{L}^{-1}$ of each analyte. (a)

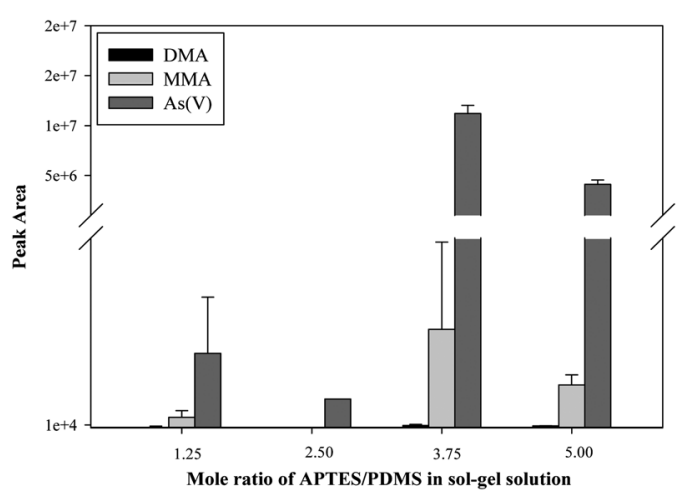

(b)

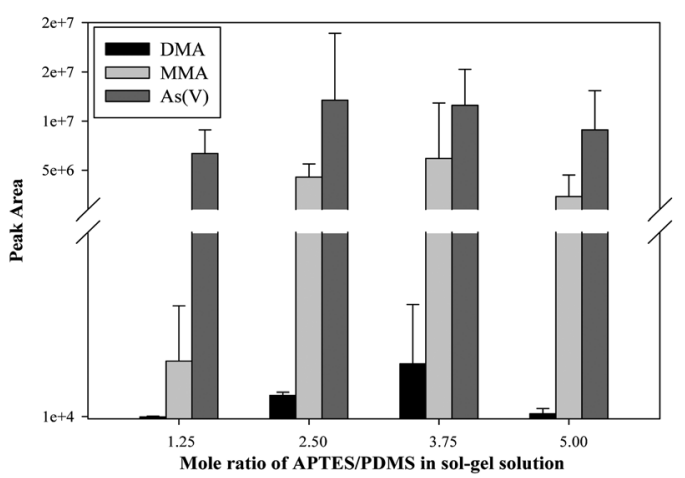

Fig. 3 Effect of mole ratio of APTES/PDMS in the sol-gel solution on extraction of the $A s(v)$ in (a) dip coated fibers, (b) electrospun coated fibers (extraction conditions; extraction time: $30 \mathrm{~min}$, As concentration: $10.0 \mu \mathrm{g} \mathrm{L}^{-1}$, solution $\mathrm{pH}$ : 5.0, stirring speed: $700 \mathrm{rpm}$, solution volume: $15 \mathrm{~mL}$, desorption conditions; desorption time: $20 \mathrm{~min}, 150 \mu \mathrm{L} 50.0 \mathrm{mM} \mathrm{KH}_{2} \mathrm{PO}_{4}$ ). Coating volumes are ca. 0.054 $\mathrm{mm}^{3}$ per fiber (dip coated) and $0.020 \mathrm{~mm}^{3}$ per fiber (electrospun coated).

Hydrolysis of the precursor, the reaction between hydrolyzed product and PDMS, and schematic representation of electrospinning set-up for SPME coating are demonstrated in Fig. 5. The amine groups in the polymeric sol-gel solution are positively charged and it can be speculated that these functional groups

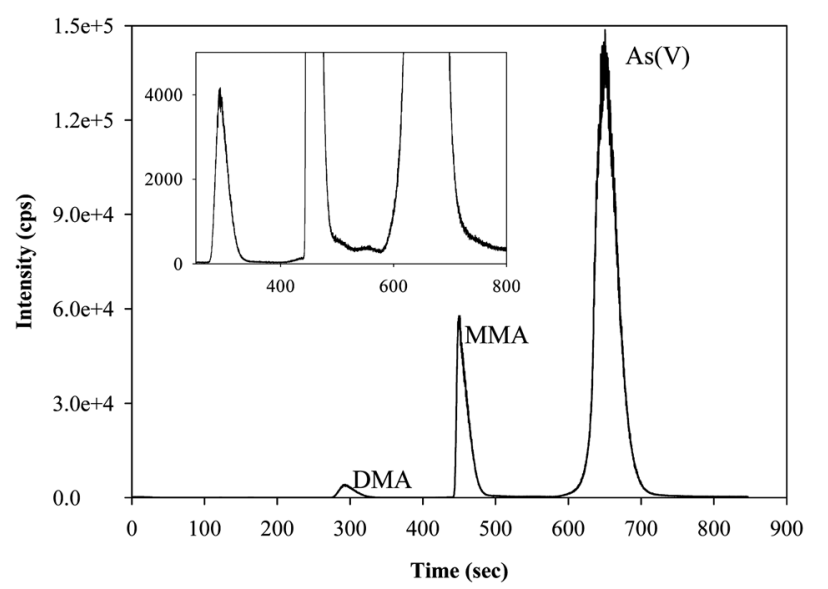

Fig. 4 A typical chromatogram obtained after application of proposed SPMEHPLC-ICPMS method (conditions are as given in Fig. 3, with electrospun coated fiber). 
(a) Hydrolysis of the precursor

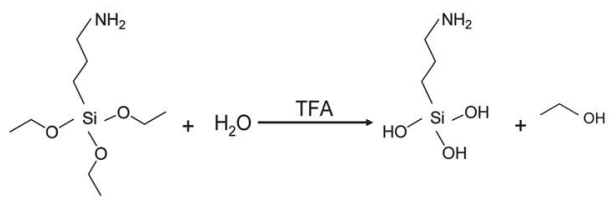

(b) The reaction between hydrolyzed product and PDMS:
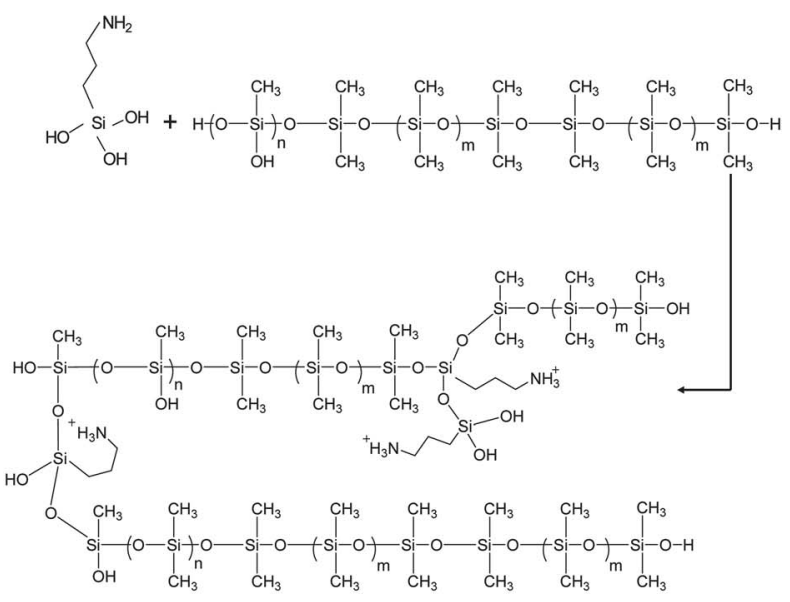

(c) SPME fiber sol-gel coating by electrospinning

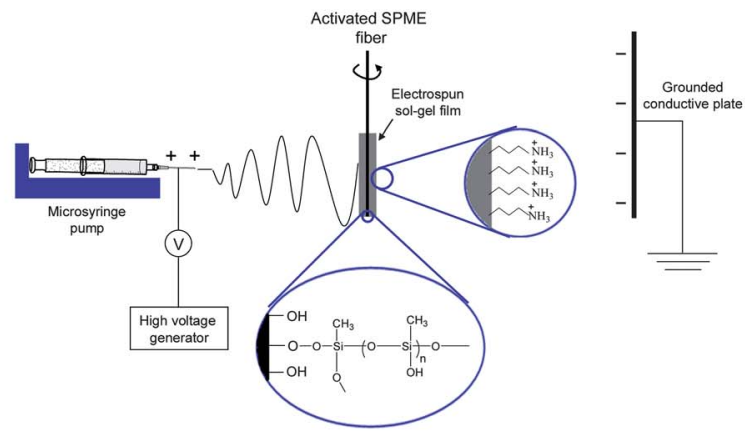

Fig. 5 Schematic diagram showing proposed design for SPME coating by combination of sol-gel chemistry and electrospinning.

which are under the influence of the electric field are oriented normal to the fiber surface. They are, therefore, more available for the extraction of arsenic species than the functional groups randomly distributed though the matrix where no electrical field is applied (dip coating method). The fibers coated by electrospinning method were used in subsequent studies.

\subsection{Optimization of extraction parameters}

3.4.1 Effect of $\mathrm{pH}$ on extraction of arsenic species. The optimization study was started with the determination of $\mathrm{pH}$ of the solution where the maximum extraction of each species was obtained. Among the tested $\mathrm{pH}$ conditions, the highest and the lowest extractions of $\mathrm{As}(\mathrm{v})$ were obtained at $\mathrm{pH} 5.0$ and 9.0, respectively. On the other hand, the extractions of MMA and DMA were particularly independent of $\mathrm{pH}$ of the solution (Fig. S2 $\dagger$ ). The coating, PDMS-weak anion exchanger, is a kind of mix-mode sorbent, and two types of interactions were expected; absorption of analytes by PDMS (nonpolar) and adsorption of analytes via electrostatic interaction through protonated amine groups. Additionally, under neutral and slightly basic pHs interaction of free amine groups with analytes should also be considered. Based on experimental findings and expected mechanisms of extractions, at neutral and lower $\mathrm{pH}$ 's the extracted amounts of the analytes depend strongly on the interaction between the weak anion exchanger surface of the SPME coating (protonated amine groups) and charge of the species. As it is well known, the weak anion exchangers are positively charged under low $\mathrm{pH}$ and are un-ionized under high $\mathrm{pH}$ conditions and this explains the reason for the lower extraction of $\mathrm{As}(\mathrm{v})$ above neutral pHs. Moreover, the correlation between the peak area and interaction of the species with the fiber shows also the tendency of the extraction. The most and the least extracted species were As(v) and DMA, correspondingly. This finding is in agreement with the elution order of the analytes from strong anion exchange column used throughout the study which also supports that the extraction is mainly through anion exchange in nature.

3.4.2 Effect of agitation time/speed on extraction of arsenic species. The effect of interaction time on extraction of the arsenic species is given in Fig. S3. $\dagger$ For DMA and MMA, increasing the contact time upto $30 \mathrm{~min}$ resulted in an increase of the extracted amounts. The extraction capability decreased if longer times were used. For As(v), the extracted amount increased gradually upto $60 \mathrm{~min}$ and decreased afterwards. A similar trend in sorption of $\mathrm{As}(\mathrm{v})$ was also observed with $\left(\mathrm{NH}_{2}\right)$ silicate in previous study. ${ }^{32}$ Results indicate that the contact time of the fiber and analyte solution is one of the most important optimization parameters. For the subsequent studies, $30 \mathrm{~min}$ was chosen as extraction time.

The effect of stirring speed of the solution during the extraction of arsenic species was also investigated. The results are given in Fig. S4. $\uparrow$ The most important outcome of the study was the inconsistency of the extracted amount of the arsenic species at various stirring speeds. According to the fundamentals of SPME, increasing the agitation speed decreases the thickness of boundary layer between coating (surface) and bulk of the solution. As a result, diffusion path decreases and this improves mass transfer. It is worth mentioning that the agitation only affects the kinetics of the extraction rather than the thermodynamics associated with the SPME process. This means that the maximum amount extracted at equilibrium will not be affected by agitation. The maximum extraction was obtained for As(v) and MMA at $700 \mathrm{rpm}$. When the solutions were stirred at $1000 \mathrm{rpm}$ extracted amount of the arsenic species were slightly decreased due to formation of vortex in the solution. The formed vortex possibly decreased the interaction of the analytes in the solution with the fiber. Another important point that should be considered is the extraction of analytes under static extraction mode. The obtained peak areas for each analyte at static extraction were comparable to each other. However the extraction of DMA decreases significantly by application of agitation. In order to explain this unexpected behavior of DMA some important aspects of SPME theory should be considered. As described in the study, two main criteria are important for the extraction of the analytes; diffusion in the sample and 
electrostatic interaction of analytes with the surface of coating. First of all, the diffusion of the analyte from bulk of the solution to the surface of coating should be evaluated. In order to explain diffusion behavior of the analytes, diffusion coefficient $(D)$ of each analyte should be considered. It is worth mentioning that the dissociation of each analyte under various pHs results in different diffusion coefficients. In the current study all analytes under consideration were able to dissociate and different form of the species can be found in the sample depending on the $\mathrm{pH}$ of solution. For instance, the neutral form of $\mathrm{As}(\mathrm{v})$ is $\mathrm{H}_{3} \mathrm{AsO}_{4}$, and it is formed under acidic conditions. As $\mathrm{pH}$ of solution increases deprotonation occurs and the other ionic species become predominant, such as $\mathrm{H}_{2} \mathrm{AsO}_{4}{ }^{-}$. With further increase of the $\mathrm{pH}, \mathrm{HAsO}_{4}{ }^{2-}$, and finally $\mathrm{AsO}_{4}{ }^{3-}$ are formed. For MMA the formed species in aqueous solution are $\mathrm{CH}_{3} \mathrm{H}_{2} \mathrm{AsO}_{3}$, $\mathrm{CH}_{3} \mathrm{HAsO}_{3}{ }^{-}, \mathrm{CH}_{3} \mathrm{AsO}_{3}{ }^{2-}$ and for DMA are $\left(\mathrm{CH}_{3}\right)_{2} \mathrm{HAsO}_{2}$ and $\left(\mathrm{CH}_{3}\right)_{2} \mathrm{AsO}_{2}{ }^{-}$(ordered from low to high pHs). The $\mathrm{pH}$ dependent distribution of species for each arsenical compound can be determined from $\mathrm{p} K_{\mathrm{a}}$ values shown in Table 2. As $\mathrm{pH}$ of the solution increases the charge on the dissociated molecule increases, which produces stronger electrostatic interaction between the dissociated molecule and water molecules and results in higher hydration level. As hydration level of a compound increases, diffusion coefficient decreases. ${ }^{33}$ Regarding the $\operatorname{As}(\mathrm{v})$ and MMA, it is possible to predict the diffusion coefficients for each analyte, such as As(v) and MMA. The extraction $\mathrm{pH}$ chosen in this study was $\mathrm{pH}$ 5.0, where the predominant species for $\mathrm{As}(\mathrm{v})$ is $\mathrm{H}_{2} \mathrm{AsO}_{4}{ }^{-}\left(D: 8.12 \times 10^{-6} \mathrm{~cm}^{2} \mathrm{~s}^{-1}\right)$ and for MMA is $\mathrm{CH}_{3} \mathrm{HAsO}_{3}{ }^{-}\left(D: 7.78 \times 10^{-6} \mathrm{~cm}^{2} \mathrm{~s}^{-1}\right) .{ }^{33}$ Although a diffusion coefficient for DMA is not reported in the aforementioned study, it can be predicted with respect to MMA. DMA is almost neutral at pH 5.0 and should have the lowest hydration level. Therefore, comparable or slightly higher diffusion coefficient for DMA can be expected compared to that of MMA. As a conclusion, in the absence of agitation (under static extraction conditions), the mass transfer rate depends on the diffusion coefficients of analytes; which is the rate determining step. Although there are some differences in diffusion coefficients of analytes, still similar extraction amount can be expected. This phenomenon can explain the similar extraction behavior of analytes observed under static extraction mode.

On the other hand, in order to explain the observed decrease on extraction of DMA by application of agitation, the affinity of analytes to coating should be taken into account. Under agitation conditions, the mass transfer is faster for all of the analytes and extraction amounts at equilibrium do not depend on diffusion in the sample. At equilibrium, analytes with high affinity to coating are extracted more than the analytes with lower affinity. Firstly, it should be mentioned that PDMS does not contribute to the extraction of analytes, and the main mechanism of extraction, as explained before, is electrostatic interaction between anionic analytes and protonated amine group (cationic). In terms of affinity of analytes to the coating, the higher extraction amount is expected for analytes with higher distribution constant $K_{\mathrm{fs}}$ (distribution of analytes between fiber and sample). In this sense and according to the extraction mechanism mentioned above, the highest extraction is expected when the coating surface is positively charged and analytes are negatively charged. The negative charge on the As(v) is higher than that of MMA and the lowest charge is on DMA. This fact supports the strongest interaction between $\operatorname{As}(\mathrm{v})$ and coating, and higher extracted amount of this species. It should be reminded that the interaction between the analytes and the coating is mainly based on adsorption. Considering the limited number of available adsorption sites on the surface, a displacement can occur. ${ }^{34}$ In this case As(v), with the strongest affinity to the coating may displace especially DMA, which has the lowest affinity to the coating. On the other hand, the displacement effect is not observed when extraction time is significantly shorter than equilibrium time. ${ }^{35}$ In the present study with a comparatively short static extraction time (30 min), no displacement effect was expected and the results of DMA under static mode and agitation conditions supported this expectation.

3.4.3 Effect of salt concentration (ionic strength) on extraction of arsenic species. The effect of $\mathrm{NaCl}$ concentration on extraction of arsenic species was studied in $1.0 \mathrm{M}, 0.10 \mathrm{M}$, and $0.010 \mathrm{M} \mathrm{NaCl}$ solutions. The results given in Fig. S5† indicate that increasing the ionic strength of the solution results in a decrease in the extracted amount of each arsenic species. It can be speculated that a decrease in the amount of the extracted analytes is related to competitive sorption of $\mathrm{Cl}^{-}$ions by protonated amine groups. The amine groups were thought to be converted into surface inactive aminium salt $\left(\mathrm{R}-\mathrm{NH}_{3}{ }^{+} \mathrm{Cl}^{-}\right)$which possibly precluded the extraction of the arsenic species in the presence of chloride ion. Since amine groups are known as weak anion exchangers, any stronger anion than arsenicals will be extracted by the coating in a competitive manner.

3.4.4 Effect of solution temperature on extraction of arsenic species. In the headspace microextraction of volatile compounds increasing the solution temperature increases the amounts of the extracted analytes ${ }^{36}$ by altering the equilibrium concentrations of analyte in the headspace and solution. In case of direct mode microextraction, a prediction of the effect of the temperature on the extraction of analyte is not easy. It depends on the exothermic or endothermic nature of the extraction as well as on the volatility of the analytes. In this study, the general trend in the effect of the solution temperature on the extraction of As(v), DMA and MMA is illustrated in Fig. S6. $\dagger$ Increasing the extraction temperature decreases the amount of extracted analytes. This finding shows the exothermic nature of the extraction of the analytes by the fibers. The effect of the temperature was more significant especially for DMA and MMA. It was observed that as the temperature was increased, the extraction decreased. For further studies, $20{ }^{\circ} \mathrm{C}$ was chosen as optimum extraction temperature.

\section{Conclusions}

In this study, the sol-gel based mixed mode (PDMS-weak anion exchanger) SPME fibers were used for direct mode extraction of DMA, MMA and As(v) from aqueous solutions followed by HPLC-ICPMS determination. Two different approaches for solgel based coating of the fibers were used, namely dip coating and electrospinning of amino functionalized sol-gel solution. 
The latter one was used for the production of sol-gel based SPME fibers. Coating experiments revealed that the fibers coated by electrospinning process showed a superior performance than dip coated fibers. The origin of this improvement is seen the alignment of PDMS chains under electrical force during the electrospinning process; thus, the further availability of pendant amino groups inevitably orientated perpendicular to the surface of the fibers. The extracted amounts of analytes were shown to be affected from various parameters: solution $\mathrm{pH}$, ionic strength, and extraction time. Due to weak anion exchange character of the coating, the optimum extraction $\mathrm{pH}$ was found to be 5.0. However, the results are promising for a relatively wide range of $\mathrm{pH}$ from 3.0 to $\mathrm{pH}$ 7.0, which show potential for various water remediation. Optimum extraction time and agitation speed were determined as $30 \mathrm{~min}$ and $700 \mathrm{rpm}$, respectively. Negative effect of the ionic strength on the extraction was observed by the addition of $\mathrm{NaCl}$ due to formation of aminium salt. Moreover, it was observed that the extraction of arsenical compounds is exothermic in nature. The proposed SPME fiber coating method can offer a very convenient way to develop efficient and selective sorbents for determination and removal of a wide range of undesirable compounds in environmental field simply by replacing the sol-gel active silane coupling reagent with another functional group. The proposed coating method is superior in terms of availability of free functional groups on surface, achieving very thin coating and homogeneous distribution of active layer on the surface. Beside the present study outlined, this novel approach can be a pioneer for further development of novel coatings and surface modifications in various fields of chemistry as well as other disciplines.

\section{Acknowledgements}

This work was financially supported by The Scientific and Technological Research Council of Turkey (TÜBITAK) through the project 108T798. The authors thank Dr Hüseyin ÖZGENER for FTIR measurements, Érica A. Souza Silva for useful discussions regarding SPME results, the Center of Material Research for SEM facility, Environmental Research Center for HPLC-ICPMS measurements at İzmir Institute of Technology, and HES Kablo for their kind donation of fiber optic cables.

\section{Notes and references}

1 Z. Y. Zhang, M. J. Yang and J. Pawliszyn, Anal. Chem., 1994, 66, A844.

2 J. Dugay, C. Miege and M. C. Hennion, J. Chromatogr., A, 1998, 795, 27.

3 K. Farhadi, R. Tahmasebi and R. Maleki, Talanta, 2009, 77, 1285.

4 T. P. Gbatu, K. L. Sutton and J. A. Caruso, Anal. Chim. Acta, 1999, 402, 67.

5 W. M. Liu, Y. A. Hu, J. H. Zhao, Y. A. Xu and Y. F. Guan, J. Chromatogr., A, 2006, 1102, 37.

6 F. Augusto, E. Carasek, R. G. C. Silva, S. R. Rivellino, A. D. Batista and E. Martendal, J. Chromatogr., A, 2010, 1217, 2533.
7 E. Turiel, J. L. Tadeo and A. Martin-Esteban, Anal. Chem., 2007, 79, 3099.

8 H. Bagheri, A. Mir and E. Babanezhad, Anal. Chim. Acta, 2005, 532, 89.

9 J. C. Wu and J. Pawliszyn, Anal. Chim. Acta, 2004, 520, 257.

10 K. P. Huang, G. R. Wang, B. Y. Huang and C. Y. Liu, Anal. Chim. Acta, 2009, 645, 42.

11 T. D. Ho, A. J. Canestraro and J. L. Anderson, Anal. Chim. Acta, 2011, 695, 18.

12 F. Zhao, Y. J. Meng and J. L. Anderson, J. Chromatogr., A, 2008, 1208, 1.

13 F. S. Mirnaghi, Y. Chen, L. M. Sidisky and J. Pawliszyn, Anal. Chem., 2011, 83, 6018.

14 N. Horzum, E. Boyaci, A. E. Eroglu, T. Shahwan and M. M. Demir, Biomacromolecules, 2010, 11, 3301.

15 N. Horzum, M. M. Demir, M. Nairat and T. Shahwan, RSC Adv., 2013, 3, 7828.

16 M. M. Demir, N. Horzum, B. Özen and S. Özçelik, J. Phys. Chem. B, 2013, 117, 10920.

17 J. W. Zewe, J. K. Steach and S. V. Olesik, Anal. Chem., 2010, 82, 5341.

18 H. Bagheri and A. Aghakhani, Anal. Methods, 2011, 3, 1284. 19 H. Bagheri and A. Aghakhani, Anal. Chim. Acta, 2012, 713, 63. $20 \mathrm{H}$. Bagheri, A. Aghakhani, M. Baghernejad and A. Akbarinejad, Anal. Chim. Acta, 2012, 716, 34.

21 G. Odian, Principles of Polymerization, John Wiley \& Sons, Inc., 2004.

22 F. Abbasi, H. Mirzadeh and A. A. Katbab, Polym. Int., 2001, 50, 1279.

23 Y. Huang, S. S. H. Ho, K. F. Ho, S. C. Lee, Y. Gao and N. S. Y. Feng, Anal. Methods, 2012, 4, 277.

24 J. C. McDonald, D. C. Duffy, J. R. Anderson, D. T. Chiu, H. K. Wu, O. J. A. Schueller and G. M. Whitesides, Electrophoresis, 2000, 21, 27.

25 D. Q. Xiao, H. Zhang and M. Wirth, Langmuir, 2002, 18, 9971. 26 A. S. M. Chong and X. S. Zhao, J. Phys. Chem. B, 2003, 107, 12650.

27 M. V. Kakade, S. Givens, K. Gardner, K. H. Lee, D. B. Chase and J. F. Rabolt, J. Am. Chem. Soc., 2007, 129, 2777.

28 J. E. Mark and B. Erman, Rubberlike Elasticity. A Molecular Primer., John Wiley \& Sons, New York, 1988.

29 M. M. Demir, B. Ozen and S. Ozcelik, J. Phys. Chem. B, 2009, 113, 11568.

30 E. H. Larsen and S. H. Hansen, Mikrochim. Acta, 1992, 109, 47.

31 M. Calatayud, J. Gimeno, D. Velez, V. Devesa and R. Montoro, Chem. Res. Toxicol., 2010, 23, 547.

32 E. Boyaci, A. Cagir, T. Shahwan and A. E. Eroglu, Talanta, 2011, 85, 1517.

33 M. Tanaka, Y. Takahashi, N. Yamaguchi, K. W. Kim, G. D. Zheng and M. Sakamitsu, Geochim. Cosmochim. Acta, 2013, 105, 360 .

34 Handbook of Solid Phase Microextraction, ed. J. Pawliszyn, Chemical Industry Press, Beijing, 2009.

35 J. Pawliszyn, J. Chromatogr. Sci., 2000, 38, 270.

36 J. Pawliszyn, Application of Solid Phase Microextraction, The Royal Society of Chemistry, Cornwall, UK, 1999. 